\title{
Non-Medical Strategies to Improve Pregnancy Outcomes of Women with Gestational Diabetes Mellitus A literature review
}

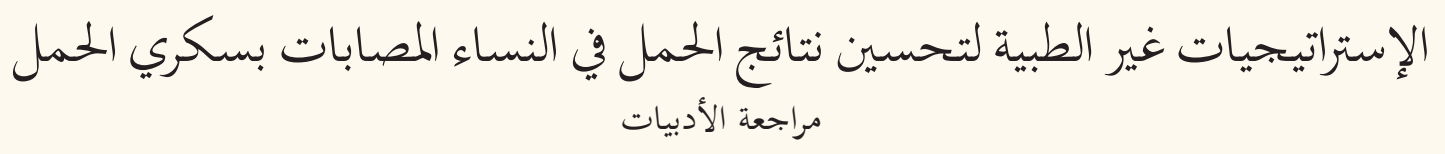

إيمان الهاشمية، كرابي ناندي، فيديا سيشان

\begin{abstract}
This review aimed to examine the literature related to non-medical strategies used to improve pregnancy outcomes of women with gestational diabetes mellitus (GDM) and to determine the risk of bias of the selected studies. Treatment for GDM is changing due to the increased prevalence of GDM-related maternal and neonatal complications. A growing body of evidence suggests that early detection, aggressive monitoring and management of GDM using non-medical strategies can greatly improve outcomes for pregnant women and their babies. PubMed ${ }^{\circledR}$ (National Library of Medicine, Bethesda, Maryland, USA), Cumulative Index to Nursing and Allied Health Literature ${ }^{\circledR}$ (EBSCO Information Services, Ipswich, Massachusetts, USA), SCOPUS ${ }^{\circledR}$ (Elsevier, Amsterdam, Netherlands) and other electronic databases were searched for relevant literature published between 2005-2015. A total of 15 studies on women with GDM that met the inclusion criteria were included in this review and assessment of risk of bias was performed for each study. The results of the studies were consistent with findings of significant improvement in maternal and neonatal outcomes when diet was combined with moderate exercise, self-monitoring of blood glucose and individualised health education. Future intervention studies in this area should be focussed on identifying and implementing factors that enhance and encourage adherence to the healthy behaviours mentioned above.
\end{abstract}

Keywords: Pregnancy Outcomes; Maternal Health; Neonatal Health; Health Behaviors; Gestational Diabetes Mellitus.

الملخص: يهدف هذا الإستعراض إلى دراسة الأدبيات المتعلقة بالإستراتيجيات غير الطبية المستخدمة لتحسين نتائج الحمل بين النساء

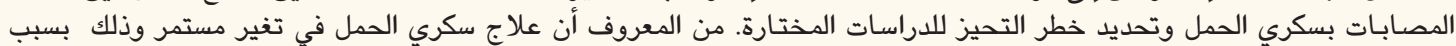

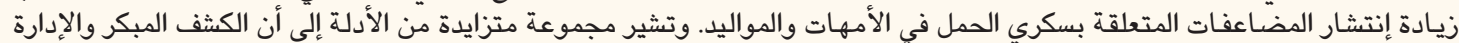

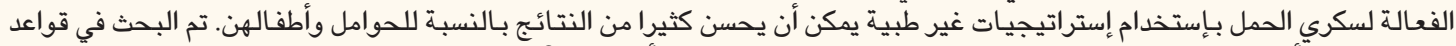

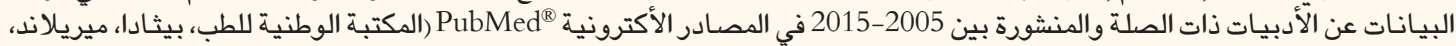

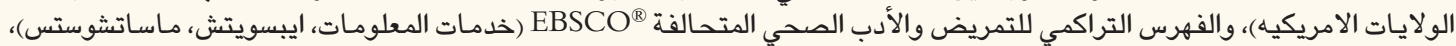
SCOPUS ${ }^{\circledR}$

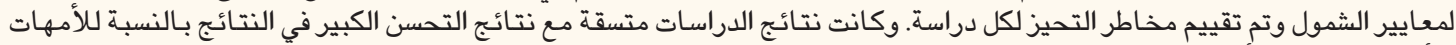

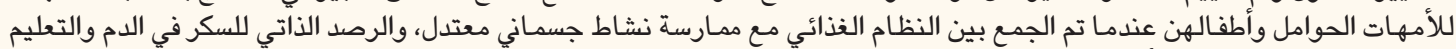



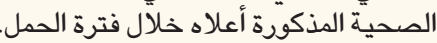
الكلمات المفتاحية: نتائج الحمل؛ صحة الأم؛ صحة حديثى الولادة؛ السلوكيات الصحية؛ سكري الحمل.

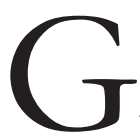
ESTATIONAL DIABETES MELLITUS (GDM) IS defined as any degree of glucose intolerance with onset or first recognition during pregnancy. ${ }^{1}$ GDM complicates about $2.7-27 \%$ of all pregnancies, varying according to a population's composition and the diagnostic criteria used. ${ }^{2,3}$ GDM has a profound association with maternal and neonatal complications. In the short term, pregnant women with GDM are at higher risk of developing pregnancy-induced hypertension and requiring a Caesarean section (CS). Moreover, women with GDM are at an increased risk of GDM in future pregnancies and also at a higher risk of developing type 2 diabetes mellitus (T2DM) in the future. ${ }^{4,5}$ GDM has also been associated with neonatal complications such as macrosomia, shoulder dystocia, birth trauma, hypoglycaemia, respiratory distress syndrome, polycythaemia and jaundice. ${ }^{3}$ Furthermore, GDM in pregnant women increases the risk of obesity and glucose intolerance in their children. ${ }^{6}$ GDM is therefore an important public health issue that has major repercussions for both mother 
and offspring. GDM is preventable and, even after onset, if detected early can be reversible. Therefore, early detection of GDM provides a window of opportunity to intervene and reduce adverse perinatal outcomes. ${ }^{\text {? }}$

The treatment of choice for GDM includes medical and non-medical interventions. Medical interventions include oral hypoglycaemic agents and insulin therapy. Although insulin therapy traditionally has been the firstline treatment, it is not without its challenges. ${ }^{8}$ Nonmedical interventions are recommended as first-line treatments and involve self-care through lifestyle modifications (typically diet and exercise) and self-monitoring of blood glucose levels (SMBG). ${ }^{9}$ Therefore, the purpose of this literature review was to investigate non-medical strategies used to treat GDM in order to improve pregnancy outcomes.

\section{Literature Review}

A literature search was performed using $\operatorname{PubMed}^{\circledR}($ National Library of Medicine, Bethesda, Maryland, USA), Cumulative Index to Nursing and Allied Health Literature $^{\circledR}$ (EBSCO Information Services, Ipswich, Massachusetts, USA) and SCOPUS ${ }^{\circledR}$ (Elsevier, Amsterdam, Netherlands) to retrieve all peer-reviewed articles published between 2005-2015. Abstracts were screened by at least two investigators using the following inclusion criteria: the article had to be published in a peer-reviewed journal between 2005-2015; written in English; involved human female subjects in the reproductive age group; and have the primary aim of assessing different strategies or interventions in decreasing the incidence of at least one GDM-related complication among women with GDM. Keywords used included "educational non-medical strategies", "improved pregnancy outcomes", "treatment", "pregnancy", "GDM", "gestational diabetes", "life-style", "diet", "exercise", "maternal outcomes" and "neonatal outcomes".

The literature search identified 15 studies that met the review's inclusion criteria [Table 1]. In the reviewed articles, the intervention group received a combination of strategies or a single strategy that was compared to a control group. Seven studies reported effects of particular dietary advice, one trial described the impact of regular exercise and five studies evaluated a combination of different lifestyle modifications involving both diet and exercise. ${ }^{10-22}$ Moreover, one study examined vitamin D supplementation and one evaluated probiotics supplementation. ${ }^{23,24}$ Insulin treatment was considered in most studies if the glycaemic target was not achieved after using other strategies. Assessment of risk of bias was performed for each study by considering eight domains: 1) randomisation of participants; 2 ) whether the study was double-blinded; 3) sample size power; 4) the use of appropriate statistical analyses; 5) an adequate intervention period; 6) managed violations in assumptions of statistical tests; 7) whether the study was controlled for confounding factors; and 8) controlled for co-intervention. Each domain was assigned one point, where a total score $\geq 5$ indicated low-to-moderate risk of bias and a total score of $<5$ indicated a moderate-to-high risk of bias.

\section{Effectiveness of Dietary Strategies}

Seven experimental studies evaluated the effectiveness of various dietary strategies-included a low-sodium diet, a low-to-moderate carbohydrate diet, a low-glycaemic index (LGI) diet and a high-fibre (HF) diet-for improving maternal and neonatal outcomes in women with GDM. ${ }^{10-16}$ Five studies implementing dietary strategies demonstrated statistically significant reductions in the incidence of at least one GDM-related complication. ${ }^{10,12,13,15,16}$

A detailed investigation of these studies revealed various outcomes. Asemi et al's 2014 randomised controlled trial (RCT) revealed that consuming a low-sodium $\operatorname{diet}(2,400 \mathrm{mg} /$ day $)$ for four weeks was associated with significantly lower CS risks and macrosomia rates and less need for insulin treatment. ${ }^{11}$ The low-sodium diet included fruits, vegetables, whole grains, low-fat dairy products and reduced amounts of saturated fats. Similarly, Landon et al. conducted a single-blinded RCT and found that a combination of dietary counselling, nutrition therapy and SMBG were significantly associated with reduced rates of pre-eclampsia, CS, macrosomia and the likelihood of babies being large for gestational age (LGA). Furthermore, the intervention group experienced significantly lower weight gain compared to the control group $(P<0.001) .{ }^{14}$ Unfortunately, specific dietary recommendations were not reported in this study.

A study with a quasi-experimental design conducted by Perichart-Perera et al. found that the risk of having a baby with low birth weight $(<2,500 \mathrm{~g})$ was significantly lower among women with GDM who had received medical nutrition intervention versus those in the control group $(P=0.041) .{ }^{16}$ Medical nutrition therapy consisted of moderate carbohydrate intake (40-45\%), SMBG and individual nutrition counselling. Compliance rates were acceptable and $73.1 \%$ of the intervention participants were adherent to the recommended dietary advice. ${ }^{16}$ A double-blinded RCT conducted by Crowther et al. reported that women with GDM who received individualised dietary advice and instructions about SMBG had a significant reduction in the incidence of serious perinatal adverse effects-including neonatal death, shoulder dystocia, bone fracture and nerve palsy-compared to women who received routine 
Table 1: Summary of selected studies evaluating the non-medical strategies used to improve pregnancy outcomes of women with gestational diabetes mellitus.

Author and
year of study

Odar et al..$^{10} \quad$ - No significant difference between intervention and control groups for requiring insulin therapy, average Apgar (2004) scoreat five minutes, rate of induction of labour, rate of CS, rate of macrosomia, rate of neonatal admission to NICU, rate of pre-eclampsia or pregnancy induced hypertension and rate of LGA $(P \geq 0.10)$.

Asemi et al. ${ }^{11} \quad$ - The DASH group had a significant reduction in CS rate, macrosomia rate and need for insulin therapy compared (2014) to the control group $(P \leq 0.01)$.

- No significant difference was found for newborn length,Apgar score at five minutes, incidence of polyhydramnios and mean gestational age $(P \geq 0.31)$.

Moreno- $\quad$ Maternal weight gain was significantly higher in the control group $(2.1 \mathrm{~kg})$ than the low-CHO group $(1.1 \mathrm{~kg} ; P=0.017)$.

Castilla et al..$^{12}$ - No significant difference was found for average gestational age at delivery, incidence of ketonuria or rates of CS, (2013) macrosomia, newborn hypoglycaemia or insulin treatment $(P \geq 0.2)$.

Crowther et al..$^{13}$ - The intervention group had a significantly higher rate of NICU admission and induction of labour compared to the (2005) control group $(P \leq 0.01)$

- No significant difference was found for neonatal death, stillbirth, neonatal bone fracture, nerve palsy, neonatal jaundice requiring phototherapy and the need for either elective or emergency CS $(P \geq 0.11)$.

Landon et al..$^{14}$ - No significant difference was found between the intervention and control groups for the average gestational age at (2009) birth, rate of neonatal jaundice, neonatal hypoglycaemia, birth trauma, preterm delivery, respiratory distress syndrome or neonatal admission to NICU $(P \geq 0.12)$.

- A significant difference was found for reduced birth weight, macrosomia rate, LGA, reduced risk for pre-eclampsia rate, reduced risk for CS, average BMI at delivery and average weight gain $(P \leq 0.02)$.

Louie et al..$^{15} \quad$ - The LGI group had a significantly lower glycaemic load than the HF group $(P \leq 0.001)$.

(2011) - No significant difference was detected for average birth weight, rate of macrosomia, rate of LGA, average maternal weight gain, rate of insulin treatment and rate of emergency CS $(P \geq 0.2)$.

Perichart- $\quad$ A significant difference was found between control and MNT groups for rate of pre-eclampsia, first maternal hospPerera et al. ${ }^{16} \quad$ italisation rate and neonatal admission rate at intermediate care unit $(P \leq 0.001)$.

(2009) - Women in the control group were at a significantly higher risk of having babies with low birth weight $(P=0.041)$.

Sugiyama et al. ${ }^{17}$ - No significant difference was found between the intervention and control groups for pregnancy-induced hyper(2014) tension, CS or the frequency of LGA and macrosomia $(P>0.05)$.

- No significant difference was found in neonatal complications (including RDS, hypoglycaemia and jaundice; $P>0.05$ ).

Barakat et al. $^{18} \quad$ - No significant difference was found between the exercise and control groups for requirement of CS, average neonatal

(2013) birth weight, average Apgar score at one minute and the average gestational age in days $(P \geq 0.2)$.

- Rate of macrosomic babies was significantly higher in the control group than the exercise group $(P=0.002)$.

Artal et al. ${ }^{19} \quad$ - 38.6\% of DR group subjects were prescribed insulin, while only $35.1 \%$ of EDR group subjects needed insulin to (2007) maintain normal blood glucose levels.

- There was no significant difference for the mean infant birth weight $(P=0.24)$.

- The incidence of CS was similar in the EDR and DR groups (50.0\% versus $44.7 \%$ ).

- Subjects in both groups who experienced weight gain were at higher risk for having macrosomic babies compared to those who had weight loss or no weight change $(P=0.12)$.

Bo et $_{\text {al }}{ }^{20} \quad$ - The DR group showed the highest maternal complications (12\%).

(2014) - Neonatal complications were highest among the DR group (10\%).

- Exercise groups (ER and BDER) had the lowest CS rates (17.7\% and 16\%, respectively).

- ER group had the lowest rate of LGA $(9.8 \%)$.

Cao et al..$^{21} \quad$. The intervention group had a significant reduction in rates of premature delivery, admission to NICU and average (2012) birth weight compared to the control group $(P \leq 0.05)$.

- Although not significant, yet subjects who received intensive treatment had a lower macrosomic rate $(P=0.143)$.

- Risk for CS was higher in the intervention group, while the risk of pre-eclampsia was higher in the control group.

- Percentage receiving insulin treatment was higher in the intervention group (33.9\% versus $26.4 \%$ ).

Yang et al. ${ }^{22} \quad$ The control group had a significantly higher rate of macrosomia and LGA than the intervention group $(P \leq 0.01)$.

(2014) - Pre-eclampsia was significantly higher in the intervention group than the control group $(P=0.031)$.

- Apgar score $<7$ was significantly higher in the control group compared to the intervention group $(P=0.02)$

Asemi et al. ${ }^{23} \quad$ - Women receiving vitamin D supplementation had a significant improvement in relation to the incidence of poly(2015) hydramnios, neonatal jaundice and newborn admission compared to the control group $(P \leq 0.04)$.

- No significant difference was found for pre-eclampsia, preterm delivery, macrosomia, need for CS, insulin treatment, babies' gestational age at delivery, Apgar scores at one and five minutes and newborn hypoglycaemia $(P \geq 0.13)$.

Lindsay et al..$^{24}$ - Probiotic supplementation had no significant effect on improving the maternal metabolic values or improving (2015) the pregnancy outcomes of women with GDM.

$C S=$ Caesarean section; $N I C U=$ neonatal intensive care unit LGA = large for gestational age; $D A S H=$ Dietary Approaches to Stop Hypertension $C H O=$ carbohydrate; $B M I=$ body mass index; $L G I=$ low-glycaemic index; $H F=$ high-fibre; $M N T=$ medical nutrition therapy; $R D S=$ respiratory distress syndrome; $D R=$ dietary recommendations only; $E D R=$ exercise and dietary recommendations; $E R=$ exercise recommendations only; $B D E R=$ behavioural dietary and exercise recommendations; $G D M=$ gestational diabetes mellitus. 
prenatal care $(P \leq 0.01) \cdot{ }^{13}$ Finally, Sugiyama et al. found a significantly lower rate of LGA and lower maternal weight gain when comparing diet therapy alone or in combination with SMBG and insulin (if needed) with the control group that received routine antenatal care. ${ }^{17}$ They found that both pre-pregnancy body mass index (BMI) and weight gain during pregnancy were independently correlated with the incidence of LGA.

Two dietary-based studies were unsuccessful in improving any maternal or neonatal outcomes of women with GDM. ${ }^{12,15}$ Moreno-Castilla et al. conducted a study in Spain that failed to find a significant difference between the low-carbohydrate group and the control diet group in terms of the maternal and neonatal outcomes. ${ }^{12}$ Similarly, Louie et al. reported no significant difference between the LGI diet group and the HF diet group in relation to the incidence of macrosomia, CS, high blood glucose levels, glycated haemoglobin (HbA1c) and the need for insulin therapy. ${ }^{15}$

Due to the fact that the reviewed studies implemented different dietary strategies, reported inconsistent results, had diverse population compositions and heterogeneous inclusion criteria, a definitive conclusion with regard to implementation of dietary strategies could not be reached. Additionally, the inclusion of pregnant women with various degrees of hyperglycaemia and the inconsistency in diagnostic criteria employed for GDM diagnosis across these studies further contributes to an inability to make informed recommendations on appropriate diets for women with GDM. For example, studies used criteria from the American Diabetes Association, the 2006 National Diabetes and Pregnancy Clinical Guidelines, World Health Organization, the Australian Diabetes in Pregnancy Society and the Society of Obstetrics and Gynecology and one study did not clearly state which diagnostic criteria was used for GDM diagnosis. ${ }^{10-12,14,15}$

Adherence to suggested dietary advice may have a considerable impact on the effectiveness of specific dietary behaviours. Only one study reported that compliance to the recommended dietary strategy resulted in improved findings. ${ }^{15}$ Perichart-Perera et al. studied a mixed population of women with GDM and women with T2DM; however, compliance with the dietary advice among the group of women with GDM was not reported, making it difficult to evaluate adherence. ${ }^{16}$ Furthermore, women's interest in the specific dietary advice that they received and their readiness to change their dietary behaviours were not measured in two unsuccessful studies, which may have played a role in the outcomes. ${ }^{12,15}$ Changing dietary behaviours may be challenging, especially during the relatively short period of time that is pregnancy. Therefore, it is essential to support and encourage women with GDM to enhance dietary adherence during the new intervention.
The quality of the evidence and the risk of bias varied across the reviewed studies. Two of the five clinical trials that reported a significant effect on improving pregnancy outcomes had a low-to-moderate risk of bias and three had a moderate-to-high risk. ${ }^{10,11,13,14,16}$ In relation to the studies that were unsuccessful in improving pregnancy outcomes in women with GDM, one had a low-to-moderate risk of bias and the other had a moderate-to-high risk..$^{12,15}$ Numerous factors may have contributed to an increased risk of bias among these studies, such as the study design, length of the study and the statistical analyses used. Two studies were not double-blinded and lacked random assignment of subjects to treatment; these findings limit the validity of the final outcomes. ${ }^{16,17}$

All five clinical trials that reported improved pregnancy outcomes had short intervention periods which may have underestimated the true impact of the interventions. ${ }^{10,11,13,14,16}$ Of these, four studies included women who were receiving insulin treatment. ${ }^{11,13,14,16}$ As insulin has a hypoglycaemic effect, this may have enhanced the effect of the intervention, therefore leading to an overestimation of positive findings. However, both clinical trials that reported the intervention group as having negative associations with improving pregnancy outcomes included women on insulin treatment which minimises the risk of insulin as a confounding factor. ${ }^{12,15}$ Furthermore, none of the studies, except Crowther et al's study, controlled for the subjects' demographic characteristics in their statistical analyses, which may have had confounding effects on the outcome and potentially increased bias. ${ }^{13}$ Finally, only two studies clearly stated that assumptions related to normality of distribution had been checked and both reported significant improvements in pregnancy outcomes. ${ }^{10,13}$

\section{Effectiveness of Exercise Strategy}

One double-blinded RCT's objective was to evaluate the effectiveness of regular exercise in reducing the risk of GDM-related complications. ${ }^{17}$ The study found that moderate-intensity resistance and aerobic exercise (three days/week, 25-30 min/session) had a significant impact on reducing macrosomia rates, decreasing the percentage of CS and restricting weight gain. More than 95\% of the subjects were adherent to the training sessions, which justifies the significant results. However, this study had a moderate-to-high risk of bias. One of the potential biases was that the group's allocation was not blinded to the project staff, thus increasing the likelihood of information bias. Moreover, no mention was made of whether statistical assumptions were checked and whether potential violations to those assumptions 
were corrected during analyses. Uncorrected violations may have led to incorrect interpretations of the final results.

\section{Effectiveness of Dietary and Exercise Strategies}

Five RCTs examined the efficacy of mixed strategies, such as diet in combination with exercise and SMBG, on improving maternal and neonatal outcomes in women with GDM and their offspring. ${ }^{18-21}$ Generally, these four studies showed a beneficial effect on limiting the incidence of at least one GDM-related complication. In a non-double-blinded RCT study, Bo et al. found that an exercise intervention applied either alone or in combination with a behavioural intervention was associated with a significant reduction in the incidence of CS, HbA1c, postprandial glucose and LGA in comparison to a diet and behavioural intervention alone. ${ }^{20}$ Behavioural strategies included oral and written cues to encourage the participant to make healthy dietary choices.

Cao et al. demonstrated that an intensive treatment regimen resulted in a significantly reduced incidence of premature delivery, neonatal admission to the neonatal intensive care unit and an average birth weight. ${ }^{21}$ Comparably, Yang et al. found that lifestyle interventions significantly reduced macrosomia rates, LGA and infant Apgar scores less than seven at one minute. ${ }^{22}$ Nevertheless, Yang et al's study showed that lifestyle interventions were significantly associated with increased risk for preeclampsia. Women who received lifestyle modifications (diet and exercise) in this study had a significantly higher rate of achieving target physical activity in the previous month (30 minutes/time, $\geq 7$ times/week) than the diet group alone; however, this finding brings to attention the risk involved in overdoing physical activities during pregnancy.

Artal et al's study showed that an exercise intervention limited weight gain among obese GDM women but there was no significant difference in gestational age at delivery, the need for CS and birth weight. ${ }^{19}$ One possible explanation is that Artal et al. considered subjects' preference in assignment to the exercise group, possibly justifying the limited weight gain within this group. On the other hand, several factors could have contributed to the non-significant findings in Artal et al's study. Missing data for the infant birth weight, gestational age at delivery and delivery method in both groups may have resulted in biased findings. However, it was found that subjects who experienced weight gain were at higher risk for having macrosomic babies in comparison to those who had weight loss or no weight change. ${ }^{18}$
It is important to note that these findings are based on limited data generated from RCTs with a moderateto-high risk of bias and caution should be taken when interpreting their results. The above studies varied in their population composition and diagnostic criteria used for GDM diagnosis. ${ }^{10-24}$ In addition, due to the nature of the pregnancies, none of these studies had an adequate intervention period, which may have led to underestimation of study findings. Four studies had a risk of bias from insulin treatment administered parallel to the study interventions, thereby affecting the validity of the results. ${ }^{13,14,16,17}$ Three studies shared the risk of information bias for not blinding outcome assessors, thereby potentially increasing the risk of inflated study findings. ${ }^{19,20,22}$ Furthermore, only Bo et al's study controlled for subjects' ages, baseline metabolic activity and pre-pregnancy BMI in their data analysis, which increases confidence in their findings. ${ }^{20}$

In light of the above limitations, the data appear to be consistent in reporting low macrosomia rates, low LGA and CS rates among women with GDM who received lifestyle modifications compared to women who received only routine antenatal care. ${ }^{20-22}$ The evidence supports the efficacy of dietary strategies combined with moderate exercise and SMBG in decreasing the incidence of GDM-related complications.

\section{Effectiveness of Dietary Supplements Strategies}

Studies that examined dietary supplements have demonstrated an impact in recommended areas of GDM outcomes. Asemi et al's double-blinded RCT showed that consumption of vitamin D supplements was significantly associated with a reduction in the incidence of polyhydramnios, neonatal jaundice and neonatal admission to the neonatal intensive care unit among women with GDM compared to patients receiving a placebo supplement. ${ }^{23}$ This study was in response to accumulating evidence towards an association between abnormal glucose levels and vitamin D deficiency. In general, this study had a lowto-moderate risk of bias. A potential source of bias was the risk for confounding factors since women in the control group may have received vitamin D from other sources, such as sun exposure and fortified foods. However, no significant difference was found between the vitamin $\mathrm{D}$ and placebo groups in relation to vitamin $\mathrm{D}$ intake. The serum 25-hydroxyvitamin D level was significantly higher among the vitamin $\mathrm{D}$ group, indicating a low risk of confounding factors. Overall, it appears that supplementing the diets of women with GDM with vitamin $\mathrm{D}$ is promising in reducing GDM-related adverse effects. 
The metabolic effects of probiotic supplementation has recently been investigated by Lindsay et al. in a doubleblinded, placebo-controlled RCT. ${ }^{24}$ This RCT revealed that probiotic supplementation did not have a significant effect on improving maternal metabolic values or improving the pregnancy outcomes for women with GDM. This could be due to the high drop-out rate (18\%) of the $\mathrm{RCT}$ after starting the treatment.

\section{Discussion}

GDM is a worldwide metabolic problem that has negative maternal and neonatal effects with long-term consequences. In light of the reviewed literature, various interventions have been offered to improve maternal and neonatal outcomes among women with GDM.

Although the majority of the reviewed articles were RCTs with powered sample sizes and appropriate statistical analyses, most of these studies had methodological issues, such as risk for confounding factors and a risk for information bias and contamination. In addition, samples lacked diversity, with the majority of these studies targeting women who are Caucasian.

As the studies in this review do not provide enough evidence to guide clinical practice and health policy; welldesigned double-blinded RCTs are needed to identify the best strategies to improve GDM outcomes. Further clinical trials should explore the type, intensity and duration of exercise that would improve pregnancy outcomes. In addition, the effectiveness of probiotics and vitamin D supplementation on pregnancy outcomes in women with GDM needs to be further studied.

\section{Conclusion}

Although the literature is growing in the field of GDM management, the incidence rate of GDM-related complications continues to rise, most likely due to unhealthy lifestyle behaviours. In general, insufficient focus has been directed towards the prevention of GDM- related complications. Furthermore, there is a lack of evidence about the strategies that enhance adherence to healthy lifestyle behaviours. The focus of upcoming research should be on identifying the factors that enhance and encourage adherence to healthy behaviours, such as increased selfefficacy towards healthy behaviours.

\section{ACKNOWLEDGEMENT}

The authors would like to thank everyone who supported the work in this paper.

\section{References}

1. World Health Organization. Diagnostic criteria and classification of hyperglycaemia first detected in pregnancy. Geneva: World Health Organization. WHO/NMH/ND/13.2. Pp. 1-62.

2. Nayak PK, Mitra S, Sahoo JP, Daniel M, Mathew A, Padma A. Feto-maternal outcomes in women with and without gestational diabetes mellitus according to the International Association of Diabetes and Pregnancy Study Groups (IADPSG) diagnostic criteria. Diabetes Metab Syndr 2013; 7:206-9. https://doi.org/10. 1016/j.dsx.2013.10.017.

3. Gasim T. Gestational diabetes mellitus: Maternal and perinatal outcomes in 220 Saudi women. Oman Med J 2012; 27:140-4. https://doi.org/10.5001/omj.2012.29.

4. Barakat MN, Youssef RM, Al-Lawati JA. Pregnancy outcomes of diabetic women: Charting Oman's progress towards the goals of the Saint Vincent Declaration. Ann Saudi Med 2010; 30:265-70. https://doi.org/10.4103/0256-4947.65253.

5. Mpondo BC, Ernest A, Dee HE. Gestational diabetes mellitus: Challenges in diagnosis and management. J Diabetes Metab Disord 2015; 14:42. https://doi.org/10.1186/s40200-015-0169-7.

6. Shriraam V, Rani MA, Sathiyasekaran BW, Mahadevan S. Awareness of gestational diabetes mellitus among antenatal women in a primary health center in South India. Indian J Endocrinol Metab 2013; 17:146-8. https://doi.org/10.4103/2230-8210.107861.

7. Mithal A, Bansal B, Kalra S. Gestational diabetes in India: Science and society. Indian J Endocrinol Metab 2015; 19:701-4. https://doi.org/10.4103/2230-8210.164031.

8. Nicholson W, Bolen S, Witkop CT, Neale D, Wilson L, Bass E. Benefits and risks of oral diabetes agents compared with insulin in women with gestational diabetes: A systematic review. Obstet Gynecol 2009; 113:193-205. https://doi.org/10.1097/AOG.0b0 $13 \mathrm{e} 318190 \mathrm{a} 459$.

9. de Holanda VR, de Souza MA, dos Santos Rodrigues MCP, Pinheiro AKB, Damasceno MMC. Knowledge of pregnant women about gestational diabetes mellitus. J Nurs UFPE Online 2012; $6: 1648-54$.

10. Odar E, Wandabwa J, Kiondo P. Maternal and fetal outcome of gestational diabetes mellitus in Mulago Hospital, Uganda. Afr Health Sci 2004; 4:9-14.

11. Asemi Z, Samimi M, Tabassi Z, Esmaillzadeh A. The effect of DASH diet on pregnancy outcomes in gestational diabetes: A randomized controlled clinical trial. Eur J Clin Nutr 2014; 68:490-5. https://doi.org/10.1038/ejcn.2013.296

12. Moreno-Castilla C, Hernandez M, Bergua M, Alvarez MC, Arce MA, Rodriguez K, et al. Low-carbohydrate diet for the treatment of gestational diabetes mellitus: A randomized controlled trial. Diabetes Care 2013; 36:2233-8. https://doi.org/10.23 $37 / \mathrm{dc} 12-2714$

13. Crowther CA, Hiller JE, Moss JR, McPhee AJ, Jeffries WS, Robinson JS, et al. Effect of treatment of gestational diabetes mellitus on pregnancy outcomes. N Engl J Med 2005; 352:2477-86. https://doi.org/10.1056/NEJMoa042973.

14. Landon MB, Spong CY, Thom E, Carpenter MW, Ramin SM, Casey B, et al. A multicenter, randomized trial of treatment for mild gestational diabetes. N Engl J Med 2009; 361:1339-48. https://doi.org/10.1056/NEJMoa0902430.

15. Louie JC, Markovic TP, Perera N, Foote D, Petocz P, Ross GP, et al. A randomized controlled trial investigating the effects of a low-glycemic index diet on pregnancy outcomes in gestational diabetes mellitus. Diabetes Care 2011; 34:2341-6. https://doi.org/10. 2337/dc11-0985.

16. Perichart-Perera O, Balas-Nakash M, Parra-Covarrubias A, Rodriguez-Cano A, Ramirez-Torres A, Ortega-González C, et al. A medical nutrition therapy program improves perinatal outcomes in Mexican pregnant women with gestational diabetes and type 2 diabetes mellitus. Diabetes Educ 2009; 35:1004-13. https://doi.org/10.1177/0145721709343125. 
17. Sugiyama T, Metoki H, Hamada H, Nishigori H, Saito M, Yaegashi N, et al. A retrospective multi-institutional study of treatment for mild gestational diabetes in Japan. Diabetes Res Clin Pract 2014; 103:412-18. https://doi.org/10.1016/j.diabres. 2013.12.017.

18. Barakat R, Pelaez M, Lopez C, Lucia A, Ruiz JR. Exercise during pregnancy and gestational diabetes-related adverse effects: A randomised controlled trial. Br J Sports Med 2013; 47:630-6. https://doi.org/10.1136/bjsports-2012-091788.

19. Artal R, Catanzaro RB, Gavard JA, Mostello DJ, Friganza JC. A lifestyle intervention of weight-gain restriction: Diet and exercise in obese women with gestational diabetes mellitus. Appl Physiol Nutr Metab 2007; 32:596-601. https://doi.org/10.1139/H07-024.

20. Bo S, Rosato R, Ciccone G, Canil S, Gambino R, Poala CB, et al. Simple lifestyle recommendations and the outcomes of gestational diabetes. A $2 \times 2$ factorial randomized trial. Diabetes Obes Metab 2014; 16:1032-5. https://doi.org/10.1111/dom.12289.
21. Cao X, Wang Z, Yang C, Mo X, Xiu L, Li Y, et al. Comprehensive intensive therapy for Chinese gestational diabetes benefits both newborns and mothers. Diabetes Technol Ther 2012; 14:1002-7. https://doi.org/10.1089/dia.2012.0142.

22. Yang $\mathrm{X}$, Tian $\mathrm{H}$, Zhang F, Zhang C, Li Y, Leng J, et al. A randomized translational trial of lifestyle intervention using a 3-tier shared care approach on pregnancy outcomes in Chinese women with gestational diabetes mellitus but without diabetes. J Transl Med 2014; 12:290. https://doi.org/10.1186/s12967-014-0290-2.

23. Asemi Z, Karamali M, Esmaillzadeh A. Favorable effects of vitamin D supplementation on pregnancy outcomes in gestational diabetes: A double blind randomized controlled clinical trial. Horm Metab Res 2015; 47:565-70. https://doi.org/10.1055/s-00 34-1394414.

24. Lindsay KL, Brennan L, Kennelly MA, Maguire OC, Smith T, Curran S, et al. Impact of probiotics in women with gestational diabetes mellitus on metabolic health: A randomized controlled trial. Am J Obstet Gynecol 2015; 212:496.e1-11. https://doi.org/10. 1016/j.ajog.2015.02.008 